\title{
Incidences and oncological outcomes of urothelial carcinoma in kidney transplant recipients
}

This article was published in the following Dove Medical Press journal: Cancer Management and Research

\author{
Jiwoong Yu \\ Chung Un Lee \\ Minyong Kang \\ Hwang Gyun Jeon \\ Byong Chang Jeong \\ Seong II Seo \\ Seong Soo Jeon \\ Hyun Moo Lee \\ Hyun Hwan Sung
}

Department of Urology, Samsung Medical Center, Sungkyunkwan University School of Medicine, Seoul, Republic of Korea
Correspondence: Hyun Hwan Sung Department of Urology, Samsung Medical Center, Sungkyunkwan University School of Medicine, 8I Irwon-ro, Gangnam-gu, Seoul 0635I, Republic of Korea

Tel +82234100737

Fax +82234106992

Email hyunhwan.sung@samsung.com
Purpose: We investigated to determine if there is an increased rate of urothelial carcinoma (UC) in kidney transplant (KT) recipients and to compare oncological outcomes of UC in KT recipients with non-KT patients.

Patients and methods: Among 2,186 patients who underwent KT in our institute, nine patients developed UC after KT in our center. Age-standardized rates (ASRs) were calculated to compare incidence rates of UC between KT patients and the general population. Additional five patients who underwent KT at other hospitals and received UC treatment at our center were included, thus a total of $14 \mathrm{KT}$ patients were compared with non-KT patients in the aspect of the treatment outcomes of bladder cancer and upper urinary tract UC (UTUC) by using generalized estimating equation (GEE).

Results: The ASRs of bladder cancer and UTUC in KT recipients were 25.5 and 129.5 times higher than that of the general population. Although there was no difference in bladder cancerspecific survival rates ( $P$-value 0.1186$)$, however, progression rates of bladder cancer were significantly higher in $\mathrm{KT}$ recipients with a relative risk of 10.53 ( $P$-value 0.0481$)$. There was no significant difference in UTUC recurrence, progression, and specific survival rate $(P$-values $0.8915,0.8806$, and 0.8116 , respectively).

Conclusion: Incidence of UC was much higher in KT recipients than the general population. Treatment outcomes for UC in KT recipients were not inferior to those of non-KT patients, except for the progression of bladder cancer. Special attention should be paid to screening and treatment of $\mathrm{UC}$ in $\mathrm{KT}$ recipients.

Keywords: urothelial carcinoma, kidney transplantation, bladder cancer, upper urinary tract urothelial carcinoma

\section{Introduction}

With the development of surgical techniques and postoperative management for kidney transplantation (KT), lifespan of recipient has prolonged and the importance of malignancy after transplantation is increasing. ${ }^{1}$ It is well known that the incidences of malignant tumors are much higher in KT recipients than in the general population. ${ }^{2-4}$ The incidences of lymphoma, lymphoproliferative disorders, and skin cancer were reported to be high in Western patients. ${ }^{5}$ The incidence of urothelial carcinoma (UC) in China and Taiwan has recently been reported to be markedly high in KT recipients. ${ }^{6,7}$ In this context, urologists are increasingly likely to encounter UC patients with transplanted kidneys. Because of differences in recipients taking immunosuppressive drugs and having a history of major surgery and nephropathy, urologists may be reluctant to apply the same treatment strategy to this group of patients as in the general population. There have been few studies about the incidence or treatment of UC in this patient

Cancer Management and Research 2019:II I57-166 
group. In this study, we investigated the incidence of UC in KT recipients and compared it with the non-KT patients. In addition, treatment strategy and treatment outcome were also compared.

\section{Patients and methods}

A retrospective cohort study was conducted at a single center and the waiver of consent was granted for retrospective data analysis such as in the current study. A total of 2,186 patients who underwent KT in our institute between February 1995 and December 2016 were investigated. Among 2,186 patients, nine patients who developed UC after KT were analyzed to calculate the incidence of UC in KT recipients. Additional five patients who underwent $\mathrm{KT}$ at other hospitals and received $\mathrm{UC}$ treatment at our center were added, thus a total of $14 \mathrm{UC}$ patients were analyzed to compare the treatment outcome between KT patients and non-KT patients. As controls for UC cases in non-KT patients, 5,264 bladder cancer patients who underwent transurethral resection of bladder tumor (TURB) or radical cystectomy and 786 upper urinary tract UC (UTUC) patients who underwent radical nephroureterectomy (NUx) in our hospital between February 1995 and December 2016 were reviewed. And these nonKT patients were matched to KT patients according to the designed matching conditions described below.

\section{Patient demographics}

The demographic data of UC patients included gender, height, and weight at diagnosis of UC, drinking and smoking status, family history of UC, hypertension, diabetes, etiology of end-stage renal disease (ESRD), date of KT surgery, KT type, postoperative immunosuppressive agents, initial clinical presentation of UC, date of UC diagnosis, UC stage, UC grade, treatments for $\mathrm{UC}$, cancer recurrence or progression, last follow-up date, and death.

\section{Analyzing and comparing the incidence of $\mathrm{UC}$ in $\mathrm{KT}$ recipients}

To calculate the cumulative incidence and age-standardized rates (ASRs; per 100,000 persons) of bladder cancer and UTUC in KT recipients, only patients who underwent KT in our institute were analyzed. The ASRs of UC from general population were obtained from the Korean Urological Cancer Statistics. ${ }^{8}$ In Korean cancer statistics, bladder neoplasms without stromal invasion (ICD10 code D09.0) were not counted as bladder cancer. Because there was no initial occurrence of bladder neoplasm without stromal invasion among our bladder cancer cases of KT recipients for incidence analysis, it was possible to directly compare the calculated
ASRs with the Korean statistics. In addition, the ratio of bladder cancer to UTUC and racial differences were compared.

\section{Analyzing and comparing treatment outcomes of $U C$ in $K T$ recipients}

After excluding patients with a history of chronic kidney disease (CKD), hemodialysis, malignancy other than UC, or receiving neoadjuvant treatment for UC from non-KT patients, matching analysis was used to compare treatment outcomes between KT patients and non-KT patients.

The matching conditions of non-KT patients were as follows: the age at diagnosis ( \pm 3 years), date of diagnosis $( \pm 3$ years), gender, T stage, grade, surgical specimen margin, and prior UC history (in the cases of later cancer). Random selection was performed for 1:2 and 1:3 matching for bladder cancer and UTUC, respectively. If there were fewer matched patients than the designed ratio, this was considered insufficient.

Treatment outcomes were investigated using different parameters, including bladder cancer progression rates, bladder cancer-specific survival rates, UTUC recurrence rates, UTUC progression rates, and UTUC-specific survival rates. Bladder cancer progression rates were defined as progression from non-muscle invasive bladder cancer (NMIBC) to muscle invasive bladder cancer (MIBC), development of UTUC after bladder cancer, and metastasis to lymph nodes or visceral organs. UTUC recurrence rates were defined as intravesical or contralateral upper urinary tract recurrence. UTUC progression rates were defined as lymph node or visceral organ metastasis. Since all deaths except one were cancer-related deaths, only comparative analysis of cancer-specific survival rates were performed.

\section{Statistical methods}

Statistical analysis was executed using SAS version 9.4 (SAS Institute Inc., Cary, NC, USA), and statistically significant differences were defined as those with $P$-values $<0.05$. Continuous variables are presented as the mean and SD, and categorical variables are presented as the number of cases and percentage. Chi-square and Fisher's exact tests were used for categorical variables, and Student's $t$-test was used for continuous variables.

The difference of ASR between the two population and the incidence ratio of bladder cancer to UTUC and male to female were compared by binomial test for one-sample proportion. And multivariate regression analysis was used to confirm the value of variables as risk factors for UC development. Generalized estimating equation (GEE; per 100,000 person-year) method was performed to evaluate the different parameters of treatment outcomes. 


\section{Results}

\section{Baseline characteristics of study patients}

Table 1 shows the baseline characteristics of 14 KT recipients with UC, including four males and ten females. Mean age at $\mathrm{KT}$ is 48.3 years and mean follow-up duration is 181.3 months. As immunosuppression regimens, steroids, cyclosporine, azathioprine, tacrolimus, and mycophenolate mofetil were used in different combinations.

Table 2 shows the characteristics of UC and treatments for each patient following KT. Bladder cancer occurred in five patients as their initial UC. UTUC occurred in nine patients: eight patients had UC in their native kidneys or ureters, and one patient developed UTUC in transplanted kidney. Average interval from the date of KT to UC diagnosis was 115.6 months.

\section{Incidence of UC in KT patients}

In the current study, the 5-year and 10-year cumulative incidence of UC in KT recipients was $0.0685 \%$ and $0.306 \%$, respectively. The ASRs of bladder cancer and UTUC in our KT recipient cohorts were 122.22 and 113.97. According to the Korean Urological Cancer Statistics, the ASRs of bladder cancer and UTUC in the general population were 4.79 and 0.88 , respectively. ${ }^{8}$ The ASRs of bladder cancer and UTUC in KT patients were 25.5 times and 129.5 times higher than the general population $(P$-value $<0.001)$.

In KT recipient cohorts, UTUC occurred twice as much as bladder cancer which is a significant difference from the general population from the Korean Urological Cancer Statistics in which bladder cancer occurred about five times more than UTUC. Furthermore, the proportion of female UC patients was higher in KT recipients; there were four male and ten female UC patients in KT recipients, whereas the proportion of male $\mathrm{UC}$ patients in the general population was 5.3 times higher.

\section{Clinicopathologic characteristics of KT recipients and matched non-KT patients}

Table 3 shows a comparison of demographic data of 1:2 matched bladder cancer patients from KT group and non-KT group. There were more bladder cancer patients from KT group who received TURB more than two times compared to matched non-KT patients. None of the KT patients was treated with Bacille Calmette-Guérin (BCG), but intravesical chemotherapies (either epirubicin or mitomycin $\mathrm{C}$ ) were performed to treat their NMIBC. All of the intravesical instillation therapy for NMIBC of non-KT bladder cancer patients were $\mathrm{BCG}$ instillation. In $\mathrm{KT}$ recipient cohorts, there were two cases of MIBC patients. Instead of radical cystectomy, bladder preservation by concurrent chemoradiotherapy (CCRTx) was performed in one case. Palliative chemotherapy was performed in another metastatic case. Radical cystectomies were performed for four out of five MIBC cases in the matched non-KT MIBC patients, and bladder preservation by CCRTx was performed for the other case. Detailed treatment courses

Table I Demographic characteristics of KT patients with UC

\begin{tabular}{|c|c|c|c|c|c|c|c|c|}
\hline $\begin{array}{l}\text { Patient } \\
\text { no. }\end{array}$ & Gender & $\begin{array}{l}\text { BMI } \\
\left(\mathrm{kg} / \mathrm{m}^{2}\right)\end{array}$ & Risk factor & $\begin{array}{l}\text { Age } \\
\text { at KT } \\
\text { (years) }\end{array}$ & $\begin{array}{l}\text { KT } \\
\text { type }\end{array}$ & $\begin{array}{l}\text { Etiology } \\
\text { of ESRD }\end{array}$ & Immunosuppressants & $\begin{array}{l}\text { F/U duration } \\
\text { after KT } \\
\text { (months) }\end{array}$ \\
\hline A & M & 30.2 & & 62 & LDKT & $\mathrm{HN}$ & Steroid, CsA, AZA & 135.8 \\
\hline B & $\mathrm{F}$ & 22.5 & & 65 & LDKT & DMCKD & FK, MMF & 72.2 \\
\hline C & $\mathrm{F}$ & 23.5 & & 54 & LDKT & Unknown & Steroid, CsA, MMF & 151.6 \\
\hline $\mathrm{D}^{\mathrm{a}}$ & $M$ & 23.9 & & 37 & DDKT & ADPKD & unknown & 35.0 \\
\hline $\mathrm{E}^{\mathrm{a}}$ & $M$ & 30.6 & Smoking & 45 & LDKT & Unknown & Steroid, CsA & 226.6 \\
\hline$F$ & $\mathrm{~F}$ & 31.1 & Smoking & 41 & DDKT & CGN & Steroid, CsA, AZA, MMF & 233.7 \\
\hline G & $\mathrm{F}$ & 22.6 & & 49 & DDKT & Unknown & Steroid, CsA, FK, MMF & 139.3 \\
\hline $\mathrm{H}$ & $\mathrm{F}$ & 23.0 & & 46 & LDKT & CGN & Steroid, CsA, MMF & 217.6 \\
\hline I & $\mathrm{F}$ & 26.6 & & 42 & DDKT & Medication & Steroid, CsA, MMF & 213.7 \\
\hline J & $\mathrm{F}$ & 24.0 & $\begin{array}{l}\text { Family history } \\
\text { of bladder } \\
\text { cancer }\end{array}$ & 53 & LDKT & $\mathrm{HN}$ & CsA, MMF & 198.5 \\
\hline K & $\mathrm{F}$ & 25.6 & & 45 & LDKT & Unknown & Steroid, CsA, MMF & 162.1 \\
\hline $\mathrm{L}^{\mathrm{a}}$ & $\mathrm{F}$ & 21.9 & & 33 & DDKT & Medication & Steroid, CsA, MMF & 315.9 \\
\hline$M^{a}$ & $\mathrm{~F}$ & 33.8 & & 50 & LDKT & CGN & Steroid, FK, MMF & 257.6 \\
\hline $\mathrm{N}^{\mathrm{a}}$ & $M$ & 21.3 & & 54 & DDKT & DMCKD & CsA & 178.6 \\
\hline Mean (SD) & & $25.7(4.0)$ & & $48.3(8.9)$ & & & & $181.3(73.3)$ \\
\hline
\end{tabular}

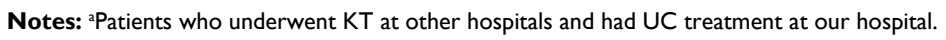

Abbreviations: ADPKD, autosomal dominant polycystic kidney disease; AZA, azathioprine; BMI, body mass index; CGN, chronic glomerulonephritis; CsA, cyclosporine A; DDKT, deceased-donor kidney transplantation; DMCKD, diabetes mellitus chronic kidney disease; ESRD, end-stage renal disease; F, female; F/U, follow-up; FK, tacrolimus; $\mathrm{HN}$, hypertensive nephropathy; KT, kidney transplantation; LDKT, living donor kidney transplantation; M, male; MMF, mycophenolate mofetil. 
Table 2 Characteristics of UC and treatment strategy

\begin{tabular}{|c|c|c|c|c|c|c|c|c|c|}
\hline $\begin{array}{l}\text { Patient } \\
\text { no. }\end{array}$ & $\begin{array}{l}\text { Age } \\
\text { at UC } \\
\text { diagnosis } \\
\text { (years) }\end{array}$ & $\begin{array}{l}\text { First } \\
\text { presentation }\end{array}$ & Initial UC type & $\begin{array}{l}\text { pT } \\
\text { stage }\end{array}$ & Grade & $\begin{array}{l}\mathbf{N} \\
\text { stage }\end{array}$ & $\begin{array}{l}\text { Treatment } \\
\text { (regimen) }\end{array}$ & $\begin{array}{l}\text { Interval } \\
\text { from KT to } \\
\text { diagnosis } \\
\text { (months) }\end{array}$ & $\begin{array}{l}\text { Alive/death } \\
\text { (survival after } \\
\text { diagnosis } \\
\text { (months)) }\end{array}$ \\
\hline$A$ & 73 & $\begin{array}{l}\text { Bowel } \\
\text { perforation }\end{array}$ & Bladder cancer & $\mathrm{T} 4$ & High & cN2 & No treatment & 135.8 & $\begin{array}{l}\text { UC-related } \\
\text { death }(0.1)\end{array}$ \\
\hline B & 70 & $\begin{array}{l}\text { Gross } \\
\text { hematuria }\end{array}$ & Bladder cancer & $\mathrm{T} 2$ & High & $\mathrm{cNI}^{\mathrm{a}}$ & $\begin{array}{l}\text { TURB } \\
+ \text { Palliative CTx. } \\
\text { (G/Cb) }\end{array}$ & 65.7 & $\begin{array}{l}\text { UC-related } \\
\text { death (7.1) }\end{array}$ \\
\hline C & 66 & $\begin{array}{l}\text { Gross } \\
\text { hematuria }\end{array}$ & Bladder cancer & $\mathrm{T} 2$ & High & cNO & $\begin{array}{l}\text { TURB + CCRTX } \\
(G / C)\end{array}$ & 146.2 & Alive (5.4) \\
\hline$D^{b}$ & 39 & $\begin{array}{l}\text { Gross } \\
\text { hematuria }\end{array}$ & Bladder cancer & TI & High & cNO & $\begin{array}{l}\text { TURB } \\
+ \text { Epirubicin } \\
\text { instillation }\end{array}$ & 16.0 & $\begin{array}{l}\text { UC-related } \\
\text { death }(21.0)\end{array}$ \\
\hline $\mathrm{E}^{\mathrm{b}}$ & 48 & Others & Bladder cancer & TI & High & cNO & TURB & 36.0 & $\begin{array}{l}\text { UC-related } \\
\text { death (195.7) }\end{array}$ \\
\hline $\mathrm{F}$ & 53 & Others & $\begin{array}{l}\text { Transplanted } \\
\text { renal pelvis/ureter }\end{array}$ & T3 & High & $\mathrm{pNx}$ & $\begin{array}{l}\text { Transplanted } \\
\text { NUx }\end{array}$ & 146.6 & Alive (88.I) \\
\hline G & 54 & $\begin{array}{l}\text { Incidental CT } \\
\text { finding }\end{array}$ & $\begin{array}{l}\text { Bilateral renal } \\
\text { pelvis }\end{array}$ & $\mathrm{T} 4$ & High & $\mathrm{pNI}$ & $\begin{array}{l}\text { Bilateral NUx } \\
+ \text { CCRTx (G/Cb) }\end{array}$ & 60.0 & $\begin{array}{l}\text { Other-cause } \\
\text { death (79.1) }\end{array}$ \\
\hline $\mathrm{H}$ & 52 & $\begin{array}{l}\text { Gross } \\
\text { hematuria }\end{array}$ & Right distal ureter & TI & High & $\mathrm{pNx}$ & Right NUx & 81.8 & Alive (I36.I) \\
\hline I & 55 & Flank pain & Right renal pelvis & T3 & High & $\mathrm{pNx}$ & Right NUx & 156.2 & Alive (57.4) \\
\hline J & 60 & $\begin{array}{l}\text { Gross } \\
\text { hematuria }\end{array}$ & Left renal pelvis & TI & High & $\mathrm{pNx}$ & Left NUx & 79.6 & Alive (I I8.9) \\
\hline K & 58 & Flank pain & $\begin{array}{l}\text { Right renal pelvis/ } \\
\text { ureter }\end{array}$ & T3 & High & $\mathrm{pNI}$ & $\begin{array}{l}\text { Rt NUx } \\
+ \text { CCRTx (G/Cb) }\end{array}$ & 157.0 & Alive (4.8) \\
\hline$L^{b}$ & 57 & Others & Left renal pelvis & $\mathrm{T} 2$ & High & pNo & Left NUx & 286.1 & Alive (29.9) \\
\hline$M^{b}$ & 61 & $\begin{array}{l}\text { Gross } \\
\text { hematuria }\end{array}$ & Right ureter & T3 & High & $\mathrm{pNx}$ & Right NUx & 122.9 & Alive (I36.0) \\
\hline $\mathrm{N}^{\mathrm{b}}$ & 65 & $\begin{array}{l}\text { Gross } \\
\text { hematuria }\end{array}$ & Left renal pelvis & $\mathrm{T} 2$ & High & $\mathrm{pNx}$ & Left NUx & 129.0 & $\begin{array}{l}\text { UC-related } \\
\text { death (5I.2) }\end{array}$ \\
\hline $\begin{array}{l}\text { Mean } \\
\text { (SD) }\end{array}$ & $57.9(9.0)$ & & & & & & & II 5.6 (67.5) & \\
\hline
\end{tabular}

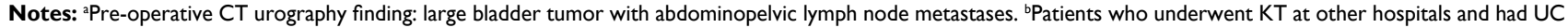
treatment at our hospital.

Abbreviations: CCRTx, concurrent chemoradiotherapy; CTx, chemotherapy; G/C/Cb, gemcitabine/cisplatin/carboplatin; KT, kidney transplantation; NUx, nephroureterectomy; TURB, transurethral resection of bladder tumor; UC, urothelial carcinoma.

for each patient are described in Figure 1. Of note, Patient C underwent bladder preservation by CCRTx for T2 bladder cancer followed by two times of TURB and showed no evidence of tumor for 5 months. In addition, Patient $E$ had no recurrence for 12 years after the first TURB. Bladder cancer recurrence was followed by visceral organ (pancreas) metastasis and death. As Table 3 shows, bladder cancer progression rates were significantly higher in KT recipients $(57.1 \%$ vs $7.1 \%$, $P$-value $=0.025)$, but there was no difference in cancer-related survival rates between the two groups.

Table 4 shows a comparison of the demographic data of 1:3 matched KT UTUC patients and non-KT patients. All patients underwent radical NUx. As shown in Figure 1, Patient F developed UC in the transplanted kidney. This case was the only UTUC in our study cohorts that occurred in a transplanted kidney. After NUx, the patient was followed up without recurrence for 94 months. Patient $\mathrm{M}$ underwent right NUx for right ureter cancer, and after recurrence in the remnant ureter, remnant ureterectomy was performed. Thereafter, no evidence of cancer was found on ureterorenoscopic biopsy of the contralateral upper urinary tract, and the patient has been disease free for 10 years. There was no significant difference in the proportion of patients who underwent adjuvant treatment. There was also no significant difference in the rates of UTUC recurrence, progression, and UTUC-specific survival.

\section{Comparison of treatment outcomes between KT patients and matched non- $\mathrm{KT}$ patients}

There were $14 \mathrm{KT}$ recipients who were treated for $\mathrm{UC}$ at our institute. One patient who died immediately after diagnosis 
Table 3 Clinicopathologic characteristics of bladder cancer cases of KT recipients and matched non-KT patients

\begin{tabular}{|c|c|c|c|c|}
\hline & & $\begin{array}{l}\text { KT patients } \\
(\mathbf{N}=7)^{\mathrm{a}}\end{array}$ & $\begin{array}{l}\text { Non-KT } \\
\text { patients }(\mathrm{N}=14)\end{array}$ & $P$-value \\
\hline Age at diagnosis (years), mean (SD) & & $59.0($ (II.8) & $58.8(I 3.1)$ & 0.971 \\
\hline Gender, n (\%) & Male & $3(42.9 \%)$ & $6(42.9 \%)$ & 1 \\
\hline Prior UTUC history, n (\%) & & $3(42.9 \%)$ & $5(35.7 \%)$ & I \\
\hline \multirow[t]{3}{*}{ T stage, $n(\%)$} & $\mathrm{Ta}$ & I (I4.3\%) & $2(14.3 \%)$ & 0.943 \\
\hline & TI & $4(57.1 \%)$ & $7(50 \%)$ & \\
\hline & $\mathrm{T} 2$ & $2(28.6 \%)$ & $5(35.7 \%)$ & \\
\hline Grade, n (\%) & High grade & $7(100 \%)$ & $14(100 \%)$ & I \\
\hline \multirow[t]{3}{*}{ TURB (\# of times), n (\%) } & 1 & I (I4.3\%) & II (78.6\%) & 0.012 \\
\hline & 2 & $2(28.6 \%)$ & $2(14.3 \%)$ & \\
\hline & $\geq 3$ & $4(57.1 \%)$ & I (7.l\%) & \\
\hline Intravesical instillation (NMIBC), n (\%) & None & I (20.0\%) & $6(66.7 \%)$ & 0.006 \\
\hline (KT patients, $\mathrm{n}=5$ & BCG & 0 & $3(33.3 \%)$ & \\
\hline matched patients, $n=9$ ) & Chemotherapy & $4(80.0 \%)$ & 0 & \\
\hline Treatment (MIBC), n (\%) & Radical cystectomy & 0 & $4(80 \%)$ & 0.103 \\
\hline (KT patients, $\mathrm{n}=2$; matched patients, $\mathrm{n}=5$ ) & Bladder preservation CCRTx & I (50\%) & I (20\%) & \\
\hline Bladder cancer progression (+), n (\%) & & $4(57.1 \%)$ & I (7.l\%) & 0.025 \\
\hline Interval from diagnosis to bladder cancer & & $42.2(67.3)$ & $55.6(76.9)$ & 0.701 \\
\hline progression (m), mean (SD) & & & & \\
\hline Cancer-related death, n (\%) & & $4(57.1 \%)$ & $2(15.4 \%)$ & 0.122 \\
\hline Follow-up duration (months), mean (SD) & & $47.7(67.3)$ & $58.0(75.9)$ & 0.765 \\
\hline
\end{tabular}

Note: ancluding patients with initial occurrence of bladder cancer and those with bladder cancer after UTUC occurrence.

Abbreviations: BCG, Bacille Calmette-Guérin; CCRTx concurrent chemoradiotherapy; KT, kidney transplantation; MIBC, muscle invasive bladder cancer; NMIBC, non-muscle invasive bladder cancer; TURB, transurethral resection of bladder tumor; UTUC, upper urinary tract urothelial carcinoma.

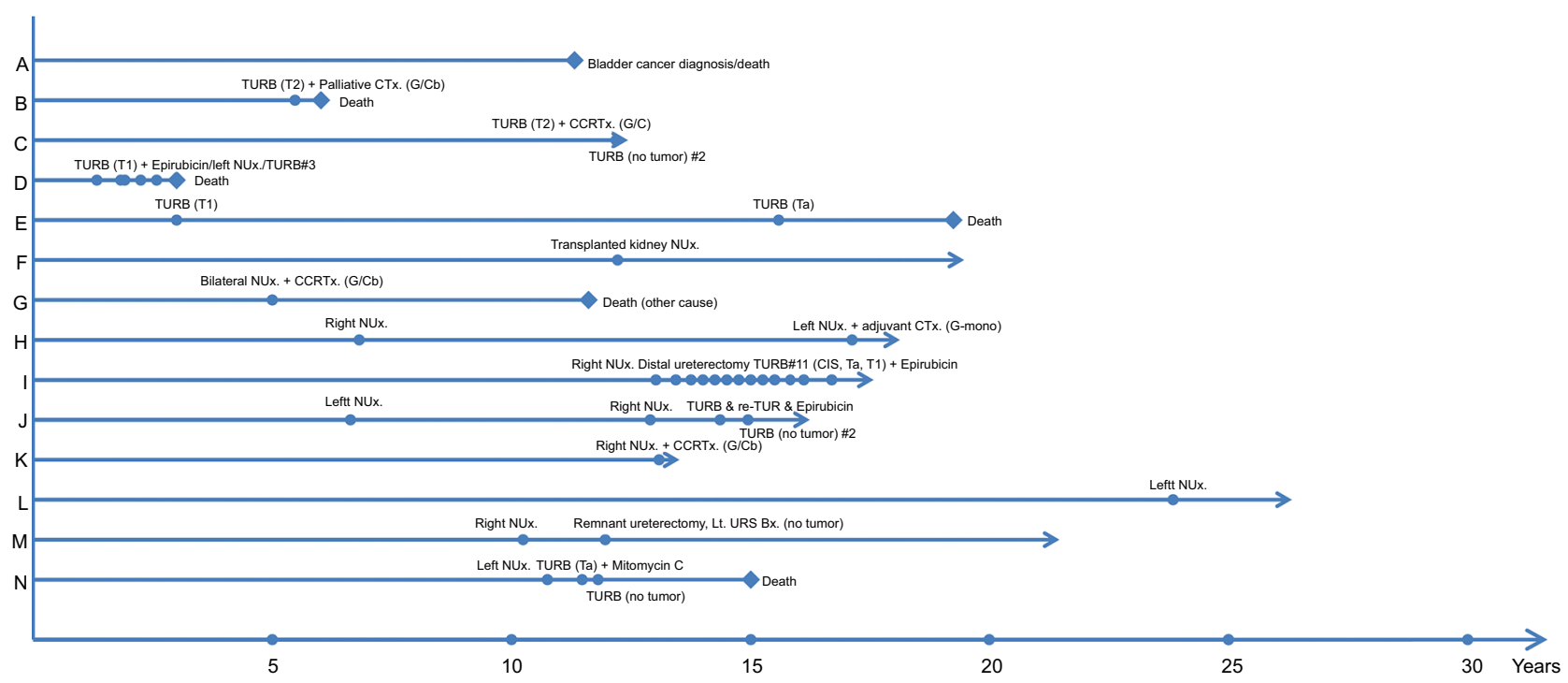

Figure I Treatment courses of $U C$ in $\mathrm{KT}$ recipients.

Abbreviations: CCRTx, concurrent chemoradiotherapy; CTx, chemotherapy; G/C/Cb, gemcitabine/cisplatin/carboplatin; G-mono, gemcitabine mono-regimen chemotherapy; NUx, nephroureterectomy; TURB, transurethral resection of bladder tumor; re-TUR, repeat-TURB.

of UC was excluded; therefore, $13 \mathrm{KT}$ recipients (eight patients from our hospital's KT cohorts and five patients who underwent KT at another hospital and had UC treatment in our hospital) were analyzed. From these $13 \mathrm{KT}$ recipients, 17 $\mathrm{UC}$ cases were analyzed to investigate treatment outcomes. This total of 17 UC cases included 13 newly developed UC cases (four bladder cancer cases and nine UTUC cases), three bladder cancer cases that subsequently developed after UTUC, and one UTUC case that subsequently developed after bladder cancer. In summary, seven bladder cancer cases and ten UTUC cases of KT recipients were analyzed to compare treatment outcomes.

The results of bladder cancer progression and bladder cancer-specific survival rate per 100,000 person-years by 
Table 4 Clinicopathologic characteristics of UTUC cases of KT recipients and matched non-KT patients

\begin{tabular}{|c|c|c|c|c|}
\hline & & $\begin{array}{l}\text { KT patients } \\
(\mathrm{N}=10)^{\mathrm{a}}\end{array}$ & $\begin{array}{l}\text { Non-KT } \\
\text { patients }(\mathrm{N}=34)\end{array}$ & $P$-value \\
\hline Age at diagnosis (years), mean (SD) & & $55.5(6.75)$ & $57.4(7.10)$ & 0.445 \\
\hline Gender, n (\%) & Male & $2(20 \%)$ & II (32.4\%) & 0.697 \\
\hline Prior bladder cancer history, n (\%) & & $\mathrm{I}(10 \%)$ & $3(8.8 \%)$ & I \\
\hline \multirow{3}{*}{ PT stage, n (\%) } & $\mathrm{Ta}$ & $\mathrm{I}(10 \%)$ & $3(8.8 \%)$ & 0.985 \\
\hline & $\mathrm{TI}-\mathrm{T} 2$ & $4(40 \%)$ & $13(38.2 \%)$ & \\
\hline & T3-T4 & $5(50 \%)$ & $18(52.9 \%)$ & \\
\hline Grade, n (\%) & High grade & $10(100 \%)$ & $34(100 \%)$ & 1 \\
\hline \multirow[t]{2}{*}{$\mathrm{N}$ stage, $\mathrm{n}(\%)$} & $\mathrm{pNx}$ & $8(80 \%)$ & 31 (9l.2\%) & 0.317 \\
\hline & $\mathrm{cNI}$ or $\mathrm{pNI}$ & $2(20 \%)$ & $3(8.8 \%)$ & \\
\hline Radical nephroureterectomy, n (\%) & & $10(100 \%)$ & $34(100 \%)$ & I \\
\hline \multirow[t]{3}{*}{ Adjuvant treatment, $\mathrm{n}(\%)$} & None & $8(80 \%)$ & $24(70.6 \%)$ & 0.007 \\
\hline & CTx & 0 & $10(29.4 \%)$ & \\
\hline & CCRTX & $2(20 \%)$ & 0 & \\
\hline \multirow[t]{2}{*}{ Adjuvant treatment, $\mathrm{n}(\%)$} & No & $8(80 \%)$ & $24(70.6 \%)$ & 0.702 \\
\hline & Yes & $2(20 \%)$ & 10 (29.4\%) & \\
\hline UTUC recurrence (+), n (\%) & & $5(50 \%)$ & $13(38.2 \%)$ & 0.716 \\
\hline Interval from diagnosis to UTUC & & $57.7(51.90)$ & $40.8(36.40)$ & 0.25 \\
\hline recurrence (months), mean (SD) & & & & \\
\hline UTUC progression (+), n (\%) & & $3(30.0 \%)$ & II (32.4\%) & 1 \\
\hline Interval from diagnosis to UTUC & & $64.0(52.38)$ & $61.8(44.50)$ & 0.897 \\
\hline progression (months), mean (SD) & & & & \\
\hline Cancer-related death, $\mathrm{m}(\%)$ & & $2(20 \%)$ & $6(17.6 \%)$ & I \\
\hline Follow-up duration (months), mean (SD) & & $71.8(47.9)$ & $71.1(38.4)$ & 0.96 \\
\hline
\end{tabular}

Notes: Including patients with initial occurrence of UTUC and those with UTUC after bladder cancer occurrence.

Abbreviations: CTx, chemotherapy; CCRTx, concurrent chemoradiotherapy; KT, kidney transplantation; UTUC, upper urinary tract urothelial carcinoma.

GEE analysis are shown in Table 5. There was no difference in bladder cancer-specific survival rates ( $P$-value 0.1186$)$; however, bladder cancer progression rates were significantly higher in KT recipients with a relative risk of 10.53 ( $P$-value 0.0481 ). As shown in Table 6 , there was no statistically significant difference in UTUC recurrence, UTUC progression, or UTUC-specific survival rate per 100,000 person-years between the two groups ( $P$-value $0.8915,0.8806$, and 0.8116 , respectively).

\section{Discussion}

As the number of KT recipients and their lifespan increases, the chance for urologists to treat $\mathrm{UC}$ in $\mathrm{KT}$ recipients increases. There may be differences and limitations in the treatment strategy of UC because of unique cancer behavior in this population and distinctive medical situations. To date, however, there have been few studies on the treatment and prognosis of this group of patients. To the best of our knowledge, the present study is the first that has attempted to match and compare the treatment outcomes of UC in KT recipients with those of the non-KT population. The pattern of UC occurrence in KT recipients was investigated along with which treatment strategies are appropriate for these patients. Furthermore, matched comparative analysis for treatment outcomes was conducted with non-KT patients using our hospital's data.

As shown in Table 1, UCs occurred an average of 115 months after KT. Similar to previous studies, UC patients from KT recipient cohorts were relatively young, and gross hematuria was the most common (50\%) initial clinical symptom. ${ }^{6,7}$ Other clinical presentations such as flank pain and incidental CT finding were noted. Several immunosuppressants were used in combination, including steroids, cyclosporine, azathioprine, tacrolimus, and mycophenolate mofetil. All patients except one had taken immunosuppressive agents including cyclosporine. There are several reports that calcineurin inhibitors (eg, cyclosporine) induce malignancy. ${ }^{9,10}$ One study suggests a less calcineurin-inhibitor-based immunosuppressant protocol for high-risk recipients. ${ }^{11}$

With regard to the incidence of $\mathrm{UC}$ in $\mathrm{KT}$ recipients compared to the general population, in this study, bladder cancer and UTUC occurred more in KT recipients than in the general population, by 25.5 times and 129.5 times, respectively. According to a study from United States, the incidence of bladder cancer after KT has been reported at around 6.5 times higher than that of the general population. ${ }^{12}$ Moreover, a study from Taiwan reported that UC occurred 398.4 times 
Table 5 Comparison of bladder cancer progression and bladder cancer-specific survival rate using GEE

\begin{tabular}{|c|c|c|c|c|}
\hline & & KT patients & Non-KT patients & $P$-value \\
\hline Bladder cancer progression rate & $\begin{array}{l}\text { Per } 100,000 \text { person-years } \\
\text { Relative risk }\end{array}$ & $\begin{array}{l}112.8 \\
10.53\end{array}$ & $\begin{array}{l}10.71 \\
1\end{array}$ & 0.0481 \\
\hline Bladder cancer-specific survival rate & $\begin{array}{l}\text { Per } 100,000 \text { person year } \\
\text { Relative risk }\end{array}$ & $\begin{array}{l}99.74 \\
4.5\end{array}$ & $\begin{array}{l}22.15 \\
1\end{array}$ & 0.1186 \\
\hline
\end{tabular}

Abbreviations: GEE, generalized estimating equation; KT, kidney transplantation.

Table 6 Comparison of UTUC recurrence, UTUC progression, and UTUC-specific survival rate using GEE

\begin{tabular}{|l|l|l|l|l|}
\hline \multicolumn{2}{|l|}{} & KT patients & Non-KT patients & P-value \\
\hline UTUC recurrence rate & Per I00,000 person year & 72.21 & 78.04 & 0.8915 \\
& Relative risk & 0.93 & 1 & \\
UTUC progression rate & Per I00,000 person year & 39.06 & 43.61 & 0.8806 \\
& Relative risk & 0.8958 & 1 & \\
UTUC-specific survival rate & Per I00,000 person year & 23.2 & 18.93 & 0.8116 \\
& Relative risk & 1.23 & 1 & \\
\hline
\end{tabular}

Abbreviations: GEE, generalized estimating equation; KT, kidney transplantation; UTUC, upper urinary tract urothelial carcinoma.

more in KT recipients. ${ }^{7}$ There are several explanations for the increased incidence of cancers in transplant recipients. Immunosuppressants cause DNA damage and may affect normal DNA repair mechanisms. ${ }^{13}$ In addition, many cancers are associated with viral infections such as BK virus after transplantation. ${ }^{14}$ Also, immune surveillance to prevent the development of malignant cells could be impaired by immunosuppressive agents. ${ }^{15}$ Also, different immune statuses result in different cancer behavior. ${ }^{16,17}$ Especially, UC is typically a cancer that affects the patients' immune system; therefore, treatment for UC with immune checkpoint inhibitors (eg, anti PD-1 or anti PD-L1) has become a new paradigm of systemic therapy for UC. ${ }^{18,19}$

Furthermore, in contrast to the general population of Korea, UTUC occurred two times more than bladder cancer in this KT recipient cohorts. As a previous report from Taiwan indicates, the incidence of UTUC is higher than that of bladder cancer, contrary to that in the general population. ${ }^{6}$ These consistent results demonstrate that there may be some vulnerable features in the upper urinary tract urothelium of Asian KT recipients. Also, in agreement with the reports from China and Taiwan, the reversed gender disparity of KT recipients compared to that of the general population was also found in our study. ${ }^{7,20}$ It is well known that, in the general population, males are three to five times more likely than females to have UC, and the Korean cancer statistics report that the proportion of male UC patients in the general population is 5.3 times higher. In contrast to this, the proportion of female UC patients was 2.5 times higher in KT recipients in the current study. We assumed that because of the geographic adjacency and racial or cultural similarities between Taiwan, China, and Korea, the markedly high incidence of UC and the predominance of female and UTUC in $\mathrm{KT}$ recipients were observed.

The treatment outcomes were compared with those of matched non-KT patients from our hospital's data. In this study, the first occurrences of bladder cancer following UTUC and UTUC after bladder cancer were counted as individual cases. The reason for this analysis was to compare posttreatment outcomes according to the location of UC. For later cancer cases, those with the same history of UC were selected for the matched controls to perform analysis. For example, with UTUC after bladder cancer, the control group was also selected for the same bladder cancer stage and grade. Also, the control patients who were selected for bladder cancer after UTUC had the same prior UTUC stage, grade, and location.

There were seven bladder cancer cases, and TURB was performed for all bladder cancer cases except one case which was diagnosed with rectal perforation. The patient underwent explorative laparotomy and expired immediately after the diagnosis. All of the initial bladder cancer cases were more than T1 stage and were high grade. Considering that bladder cancers in immunocompromised host such as HIV-infected patients have aggressive pathological features, immunosuppressants taken by transplant patients affect the development of aggressive cancer. ${ }^{21}$ Intravesical chemotherapies (either epirubicin or mitomycin C) were performed in four cases out of five NMIBC cases from KT patients. Of note, BCG, a live attenuated form of Mycobacterium bovis, instillation was not performed in KT recipients. Because of the theoretically high risk of side 
effects such as sepsis in immunocompromised patients, BCG instillation is contraindicated in transplant recipients. However, there are a few case reports indicating that BCG instillation was performed without complication in such patients. ${ }^{22,23}$ According to our matching analysis comparing the treatment outcomes of bladder cancer, the progression rates of bladder cancer were significantly higher in $\mathrm{KT}$ recipients. We thought this high rate of progression is related to the lack of BCG use and further research is needed on BCG instillation therapy for NMIBC in transplant recipients. Two cases of MIBC from KT patients exist; one patient underwent bladder preservation by CCRTx for T2 bladder cancer with curative intent followed by two times of TURB for 5 months, and the patient has been with no evidence of tumor. Another MIBC patient with metastatic disease underwent palliative chemotherapy. Although no patient underwent radical cystectomy for MIBC in this study, there were several studies that recommend cystectomy for MIBC in KT recipients. ${ }^{24-26}$ In matching analysis, bladder cancer-specific survival rates were not significantly different than those of non-KT patients.

NUx was performed for all patients with UTUC. There was only one case of UTUC occurring in the transplanted renal pelvis, and the other cases occurred in the native kidney and ureter. After removal of the transplanted kidney and ureter, the patient with UC in the transplanted renal pelvis was disease-free for 94 months. Because this patient received the graft from deceased donor, we could not investigate the occurrence of malignancy in the donor's other kidney and ureter. Only a few case reports exist, the occurrence of UTUC in kidney grafts is a very rare phenomena. ${ }^{11,27}$ No additional treatment was performed for $\mathrm{T} 1$ cancer, but patients with metastatic disease were treated with adjuvant treatment. For chemotherapy, one patient received gemcitabine mono-regimen, and the other two patients received combinations of gemcitabine and carboplatin, instead of cisplatin, which has minimal nephrotoxicity. Among nine UTUC patients, three patients (33.3\%) eventually had UTUCs in their bilateral native kidneys. In addition, patient $\mathrm{G}$ in Figure 1 underwent bilateral NUx followed by CCRTx then survived for more than 5 years and died due to reasons other than UC. From China, there are several reports of synchronous cancers in bilateral upper urinary tracts that were confirmed in around $40 \%$ of patients. ${ }^{6,7,28}$ These studies also recommend considering prophylactic contralateral NUx in KT recipients with unilateral UTUC. After matching clinicopathologic characteristics of patients, all outcome parameters of UTUC in KT recipients were comparable with those of non-KT patients. To detail, there was only one case which the UTUC developed after bladder cancer. The later UTUC patient experienced UTUC recurrence and progression and eventually died because of UC. In contrast to that, the matched three non-KT UTUC patients experienced only cancer recurrences, and all these three patients were progression-free and alive till the study time. We suggested that NUx should be the first consideration for UTUC in KT recipients. In addition to that, as in the general population, carboplatin, rather than cisplatin, may be an appropriate alternative chemotherapeutic agent for treating UTUC in KT recipients. ${ }^{29}$

In our hospital's transplant center, pre-transplant urological evaluation includes routine urinalysis, urine microscopy, urine protein/creatinine ratio, voiding cystourethrography, and kidney ultrasonography. After transplantation, there is no routine urologic evaluation of $\mathrm{KT}$ recipients. This present study suggested that a regular urologic assessment such as urine cytology or cystoscopy is needed. Also, the higher incidence, especially in the female $\mathrm{KT}$ recipient of UC in other races, should be evaluated in a further multicenter study.

There were several limitations of this study. First, it was a nonrandomized retrospective cohort study. The patient data maybe incomplete. We cannot rule out the possibility of residual confounding variables of measured or unmeasured factors. Second, an incidence study from a single center population does not represent all KT recipients due to selection bias. Third, analyzing the treatment outcomes of subsequently developed UC cases overlapped with other cases. However, the strengths of this study are that the power was improved by performing elaborate matching analysis, including the clinicopathologic history of UC in subsequently developed UC cases, and the mean follow-up period was relatively long at 181 months.

\section{Conclusion}

The incidence of UC was much higher in KT recipients compared to that of the general population, especially for female KT recipients. Furthermore, the proportion of UTUC was much higher than the proportion of bladder cancer. According to our matching analysis, active treatment including surgical and systemic therapies for UC in $\mathrm{KT}$ recipients results in outcomes that are not inferior to those of non-KT patients in our hospital. Special attention should be paid to $\mathrm{UC}$ in $\mathrm{KT}$ recipients, including urologic screening, and active treatment could benefit to these patients. 


\section{Ethical approval}

This study was approved by the institutional review board at Samsung Medical Center and abided with the Declaration of Helsinki. For this type of study, formal consent was not required and data were anonymous.

\section{Acknowledgment}

The authors thank Statistics and Data Center at Samsung Medical Center for their statistical support. The abstract of this paper was presented at the 2018 American Urological Association (AUA) Annual Meeting as a poster presentation with interim findings. The poster's abstract was published in "Poster Abstracts" in The Journal of Urology (https://doi. org/10.1016/j.juro.2018.02.211).

\section{Author contributions}

HHS, HML, SSJ, and JY designed the study. SIS, BCJ, HGJ, and CUL planned the study and collected the clinical data. MK and JY analyzed the data. JY, MK, HGJ, and BCJ wrote the early draft of the manuscript, and JY and HHS updated and revised the final draft of the manuscript. All authors contributed to data analysis, drafting and revising the article, gave final approval of the version to be published, and agree to be accountable for all aspects of the work.

\section{Disclosure}

The authors report no conflicts of interest in this work.

\section{References}

1. Mazuecos A, Muñoz Terol JM, García Alvárez T, et al. Increase in malignancies as cause of death in renal transplant patients. Transplant Proc. 2009;41(6):2159-2162.

2. Penn I. Occurrence of cancers in immunosuppressed organ transplant recipients. Clin Transpl. 1998;147-158.

3. Buzzeo BD, Heisey DM, Messing EM. Bladder cancer in renal transplant recipients. Urology. 1997;50(4):525-528.

4. Hevia V, Gómez V, Díez Nicolás V, et al. Development of urologic de novo malignancies after renal transplantation. Transplant Proc. 2014;46(1):170-175.

5. Penn I. Cancers in renal transplant recipients. Adv Ren Replace Ther. 2000;7(2):147-156.

6. Kao YL, Ou YC, Yang CR, Ho HC, Su CK, Shu KH. Transitional cell carcinoma in renal transplant recipients. World J Surg. 2003;27(8): 912-916.

7. Wu MJ, Lian JD, Yang CR, et al. High cumulative incidence of urinary tract transitional cell carcinoma after kidney transplantation in Taiwan. Am J Kidney Dis. 2004;43(6):109 -1097.

8. Joung JY, Lim J, Oh CM, et al. Current Trends in the Incidence and Survival Rate of Urological Cancers in Korea. Cancer Res Treat. 2017;49(3): 607-615.

9. Penn I, First MR. Development and incidence of cancer following cyclosporine therapy. Transplant Proc. 1986;18(2 Suppl 1):210-215.
10. Vogt P, Frei U, Repp H, Bunzendahl H, Oldhafer K, Pichlmayr R Malignant tumours in renal transplant recipients receiving cyclosporin: survey of 598 first-kidney transplantations. Nephrol Dial Transplant. 1990;5(4):282-288.

11. Hong YA, Hwang HS, Sul HJ, Kim SY, Chang YK. Transitional cell carcinoma involving graft kidney in a kidney transplant recipient: a case report. BMC Nephrol. 2017;18(1):299.

12. Cox J, Colli JL. Urothelial cancers after renal transplantation. Int Urol Nephrol. 2011;43(3):681-686.

13. Gutierrez-Dalmau A, Campistol JM. Immunosuppressive therapy and malignancy in organ transplant recipients: a systematic review. Drugs. 2007;67(8):1167-1198.

14. YinWY,Lee MC,LaiNS, Lu MC. BK virus as a potential oncovirus for bladder cancer in a renal transplant patient. J Formos Med Assoc. 2015;114(4): 373-374.

15. Bustami RT, Ojo AO, Wolfe RA, et al. Immunosuppression and the risk of post-transplant malignancy among cadaveric first kidney transplant recipients. Am J Transplant. 2004;4(1):87-93.

16. Fidler IJ, Gersten DM, Kripke ML. Influence of immune status on the metastasis of three murine fibrosarcomas of different immunogenicities. Cancer Res. 1979;39(10):3816-3821.

17. Fidler IJ, Gersten DM, Riggs CW. Relationship of host immune status to tumor cell arrest, distribution, and survival in experimental metastasis. Cancer. 1977;40:46-55.

18. Chism DD. Urothelial Carcinoma of the Bladder and the Rise of Immunotherapy. J Natl Compr Canc Netw. 2017;15(10):1277-1284.

19. Gupta S, Gill D, Poole A, Agarwal N. Systemic Immunotherapy for Urothelial Cancer: Current Trends and Future Directions. Cancers (Basel). 2017;9(2):E15.

20. Li XB, Xing NZ, Wang Y, et al. Transitional cell carcinoma in renal transplant recipients: a single center experience. Int J Urol. 2008;15(1): 53-57.

21. Chawki S, Ploussard G, Montlahuc C, et al. Bladder cancer in HIVinfected adults: an emerging issue? Case-reports and systematic review. PLoS One. 2015;10(12):e0144237.

22. Neuzillet Y, Davin JL, Lechevallier E. Intravesical BCG instillations for the treatment of superficial bladder tumours in renal transplant recipients. Prog Urol. 2006;16(1):32-35.

23. Swietek N, Waldert M, Susani M, Schatzl G, Klatte T. Intravesical bacillus Calmette-Guérin instillation therapy for non-muscle-invasive bladder cancer following solid organ transplantation. Wien Klin Wochenschr. 2013;125(7-8):189-195.

24. Giessing M, Türk I, Schoenberger B, Loening SA. Orthotopic neobladder after kidney transplantation in a male patient with recurring urothelial carcinoma and renal cancer. J Urol. 2001;166(4):1383.

25. Shirakawa H, Ishida H, Hashimoto Y. Continent orthotopic ileal neobladder after kidney transplantation in a patient with urothelial cell carcinoma associated with Chinese herb nephropathy. Transplant Proc. $1741 ; 2008: 40$

26. Master VA, Meng MV, Grossfeld GD, Koppie TM, Hirose R, Carroll PR. Treatment and outcome of invasive bladder cancer in patients after renal transplantation. J Urol. 2004;171(3):1085-1088.

27. Hevia V, Gómez V, Alvarez S, et al. Transitional cell carcinoma of the kidney graft: an extremely uncommon presentation of tumor in renal transplant recipients. Case Rep Transplant. 2013;2013:1-5.

28. Tian Y, Hou HJ, Guo YW, et al. [Necessity of prophylactic contralateral nephroureterectomy in the renal transplantation patients with transitional cell carcinoma of unilateral upper urinary tract]. Beijing Da Xue Xue Bao Yi Xue Ban. 2013;45(4):558-561. Chinese.

29. Necchi A, Pond GR, Raggi D, et al. Efficacy and safety of gemcitabine plus either taxane or carboplatin in the first-line setting of metastatic urothelial carcinoma: a systematic review and meta-analysis. Clin Genitourin Cancer. 2017;15(1):23-30.e2. 


\section{Publish your work in this journal}

Cancer Management and Research is an international, peer-reviewed open access journal focusing on cancer research and the optimal use of preventative and integrated treatment interventions to achieve improved outcomes, enhanced survival and quality of life for the cancer patient. The manuscript management system is completely online and includes a very quick and fair peer-review system, which is all easy to use. Visit http://www.dovepress.com/testimonials.php to read real quotes from published authors.

Submit your manuscript here: https://www.dovepress.com/cancer-management-and-research-journal 